\title{
Syntheses, Characterization, and Luminescence Properties of Four Metal-Organic Frameworks Based on a Linear-Shaped Rigid Pyridine Ligand
}

Zemin Ju, Wei Yan, Xiangjing Gao, Zhenzhen Shi, Ting Wang and Hegen Zheng*

State Key Laboratory of Coordination Chemistry, School of Chemistry and Chemical Engineering, Collaborative Innovation Center of Advanced Microstructures, Nanjing University, Nanjing 210093, P. R. China.

\section{Experimental section}

Synthesis of compound $\left\{[\mathrm{Cd}(\mathrm{DPBT})(\mathrm{BDC})] \cdot 2\left(\mathrm{H}_{2} \mathrm{O}\right)\right\}_{n} \quad(1)$. A mixture of $\mathrm{Cd}\left(\mathrm{NO}_{3}\right)_{2} \cdot 4 \mathrm{H}_{2} \mathrm{O}$ (30.8 mg, $\left.0.1 \mathrm{mmol}\right)$, BDC (16.6 mg, $\left.0.1 \mathrm{mmol}\right)$, and DPBT (29.0 mg, $0.1 \mathrm{mmol}$ ) was dissolved in mixed solution consisting of $\mathrm{H}_{2} \mathrm{O}(3 \mathrm{ml})$, DMF (1 ml) and acetonitrile $(0.5 \mathrm{ml})$. The reaction mixture was sealed in a PTFE-lined stainless-steel vessel under autogenous pressureand heated at $100^{\circ} \mathrm{C}$ for 3 days. Large quantities of yellow lump crystals were obtained after cooling, and crystals were filtered off, washed with quantities of distilled water, and dried under ambient conditions. The yield of the reaction was ca. 47\% (based on DPBT ligand). Calcd for $\mathrm{C}_{24} \mathrm{H}_{18} \mathrm{SN}_{4} \mathrm{O}_{6} \mathrm{Cd}: \mathrm{C}, 47.81 \%$; H, 3.05\%; N, 9.29\%. Found: C, 47.83\%; H, 3.02\%; N, 9.32\%.Main infrared spectral data (KBr, $\left.\mathrm{cm}^{-1}\right)$ : 3421 (s), 3052 (w), 2929(w), 1608 (m), 1566(s), 1387 (s), 1218 (w), 1021 (w), 810 (m), 752 (m), 522 (w), 514 (m).

Synthesis of compound $\left\{\left[Z_{n_{2}}(\mathrm{DPBT})_{2}(\mathrm{IPA})_{2}\right] \cdot 2(\mathrm{DMA})\right\}_{n}(2)$. A mixture of $\mathrm{Zn}\left(\mathrm{NO}_{3}\right)_{2} \cdot 6 \mathrm{H}_{2} \mathrm{O}(29.7 \mathrm{mg}, 0.1 \mathrm{mmol})$, BDC (16.6 mg, $\left.0.1 \mathrm{mmol}\right)$, and DPBT (29.0 mg, $0.1 \mathrm{mmol}$ ) was dissolved in mixed solution consisting of $\mathrm{H}_{2} \mathrm{O}(3 \mathrm{ml})$ and DMA (1 ml). The reaction mixture was sealed in a PTFE-lined stainless-steel vessel under 
autogenous pressure and heated at $100{ }^{\circ} \mathrm{C}$ for 3 days. Large quantities of yellow crystals were obtained after cooling, and crystals were filtered off, washed with quantities of distilled water, and dried under ambient conditions. The yield of the reaction was ca. $76 \%$ (based on DPBT ligand). Calcd for $\mathrm{C}_{56} \mathrm{H}_{46} \mathrm{~S}_{2} \mathrm{~N}_{10} \mathrm{O}_{10} \mathrm{Zn}_{2}$ : C, 55.41\%; H, 3.82\%; N, 11.53\%. Found: C, 55.39\%; H, 3.84\%; N, 11.55\%.Main infrared spectra data (KBr, cm${ }^{-1}$ ): 3420 (s), 3059 (w), 2929 (w), 1620 (s), 1553 (m), 1390 (s), 1226 (w), 1070 (w), $1021(w), 817$ (m), 727 (s).

Synthesis of compound $\left\{\left[\mathrm{Co}_{2}(\mathrm{DPBT})_{2}(\mathrm{BDC})_{2}\right] \cdot \mathbf{2}(\mathrm{DMF}) \cdot \mathbf{5}\left(\mathrm{H}_{2} \mathrm{O}\right)\right\}_{n}(3)$. A mixture of $\mathrm{Co}\left(\mathrm{NO}_{3}\right)_{2} \cdot 6 \mathrm{H}_{2} \mathrm{O}$ (29.1 mg, $\left.0.1 \mathrm{mmol}\right)$, BDC (16.6 mg, $\left.0.1 \mathrm{mmol}\right)$, and DPBT (29.0 mg, $0.1 \mathrm{mmol}$ ) was dissolved in mixed solution consisting of $\mathrm{H}_{2} \mathrm{O}(3 \mathrm{ml})$ and DMF (1 $\mathrm{ml}$ ). The reaction mixture was sealed in a PTFE-lined stainless-steel vessel under autogenous pressure and heated at $100{ }^{\circ} \mathrm{C}$ for 3 days. Large quantities of red crystals were obtained after cooling, and crystals were filtered off, washed with quantities of distilled water, and dried under ambient conditions. The yield of the reaction was ca. 76\% (based on DPBT ligand). Calcd for $\mathrm{C}_{54} \mathrm{H}_{52} \mathrm{~S}_{2} \mathrm{~N}_{10} \mathrm{O}_{15} \mathrm{Co}_{2}$ : C, 51.35\%; $\mathrm{H}, 4.15 \%$; $\mathrm{N}$, 11.08\%. Found: C, 51.34\%; H, 4.17\%; N, 11.10\%.Main infrared spectra data (KBr, $\mathrm{cm}^{-1}$ ): 3420 (s), 3059 (w), 2921 (w), 1649 (s), 1553 (m), 1398 (s), 1218 (w), 1076 (w), $1024(\mathrm{w}), 825(\mathrm{~m}), 728(\mathrm{~s})$.

Synthesis of compound $\left\{\left[\mathrm{Ni}(\mathrm{DPBT})_{2}\left(\mathrm{H}_{2} \mathrm{O}\right)_{4}\right] \cdot(\mathrm{BDC})\right\} \quad(4)$. A mixture of $\mathrm{Ni}\left(\mathrm{NO}_{3}\right)_{2} \cdot 6 \mathrm{H}_{2} \mathrm{O}(29.1 \mathrm{mg}, 0.1 \mathrm{mmol})$, BDC (16.6 mg, $\left.0.1 \mathrm{mmol}\right)$, and DPBT (29.0 mg, $0.1 \mathrm{mmol}$ ) was dissolved in mixed solution consisting of $\mathrm{H}_{2} \mathrm{O}(3 \mathrm{ml})$ and DMF (1 ml). The reaction mixture was sealed in a PTFE-lined stainless-steel vessel under autogenous pressure and heated at $100{ }^{\circ} \mathrm{C}$ for 3 days. Large quantities of green crystals were obtained after cooling, and crystals were filtered off, washed with quantities of distilled water, and dried under ambient conditions. The yield of the reaction was ca. $76 \%$ (based on DPBT ligand). Calcd for $\mathrm{C}_{40} \mathrm{H}_{32} \mathrm{~S}_{2} \mathrm{~N}_{8} \mathrm{O}_{8} \mathrm{Ni}$ : C, 54.87\%; H, 3.68\%; N, 12.80\%. Found: C, 54.85\%; H, 3.70\%; N, 12.79\%.Main infrared spectra data (KBr, cm $\left.{ }^{-1}\right)$ : 3425 (s), 3062 (w), 2919 (w), 1637 (s), 1572 (m), 1402 (s), 1228 (w), $1086(\mathrm{w}), 1016(\mathrm{w}), 820(\mathrm{~m}), 743(\mathrm{~s})$.

Materials and methods. All commercially available chemicals were of reagent 
grade and used as received without further purification. IR absorption spectra of the compounds were recorded in the range of $400-4000 \mathrm{~cm}^{-1}$ on a Nicolet (Impact 410) spectrometer with $\mathrm{KBr}$ pellets. $\mathrm{C}, \mathrm{H}$ and $\mathrm{N}$ analyses were carried out with a Perkin-Elmer model 240C elemental analyzer. Thermogravimetric analyses (TGA) were performed on a Perkin-Elmer thermal analyzer under nitrogen with a heating rate of $10{ }^{\circ} \mathrm{C} \mathrm{min}^{-1}$. Powder X-ray diffraction (PXRD) patterns were collected in the $2 \theta=5-50^{\circ}$ range with a scan speed of $0.1 \mathrm{~s} \mathrm{deg}^{-1}$ on a Bruker D8 Advance instrument using $\mathrm{Cu} \mathrm{Ka}$ radiation at room temperature. The calculation was performed in the Gaussian 09 program package. ${ }^{1}$ Without any symmetrical constraints, the geometrical structure of DPBT was optimized by the DFT-B3LYP/LanL2MB level (a semiempirical method). ${ }^{2}$ Incorporating the optimized model in the ZINDO method, ${ }^{3}$ we calculated excitation energies of DPBT and nitro compounds.

Crystal structure determination. Single crystals of 1-4 were prepared by the methods described in the synthetic procedure. X-ray crystallographic data of 1-4 were collected at room temperature using epoxy-coated crystals mounted on glass fiber. X-ray crystallographic data of these complexes were collected on a Bruker Apex Smart CCD diffractometer with graphite-monochromated Mo-Ka radiation ( $\lambda=$ $0.71073 \AA$ ). Structure solutions were solved by direct methods, and the non-hydrogen atoms were located from the trial structure and then refined anisotropically with SHELXTL using full-matrix least-squares procedures based on F2 values. ${ }^{4}$ The hydrogen atom positions were fixed geometrically at calculated distances and allowed to ride on the parent atoms. The DMA molecules of compound 2 and the water and DMF molecules of compound 3 were highly disordered and could not be modeled properly, thus the SQUEEZE routine of PLATON was applied to remove the contributions to the scattering from the solvent molecules. The numbers of solvent molecules in compounds 2 and $\mathbf{3}$ were obtained by element analyses. Compounds 1-4 crystal data and structure refinement parameters are listed in Table S1, and the main bond lengths $(\AA)$ and bond angles ( ${ }^{\circ}$ ) are listed in Table S2.

PL measurements. The PL (photoluminescence) properties of compounds 1 and 2 were investigated in the solid state and in different solvents emulsions at room 
temperature. The emulsions were prepared by introducing $1 \mathrm{mg}$ of $\mathbf{1}$ or $\mathbf{2}$ powder into $3.00 \mathrm{~mL}$ of solvent and then completing ultrasonic agitation for $30 \mathrm{~min}$, and standing at room temperature for three days before testing. Fluorescent spectra were recorded with a SHIMAZU VF-320 X-ray fluorescence spectrophotometer at room temperature.

Table S1. Crystallographic Data and Structure Refinement Details for Compounds

1-4.

\begin{tabular}{|l|l|l|l|l|}
\hline Compounds & $\mathbf{1}$ & $\mathbf{2}$ & $\mathbf{3}$ & $\mathbf{4}$ \\
\hline Formula & $\mathrm{C}_{24} \mathrm{H}_{18} \mathrm{CdN}_{4} \mathrm{O}_{6} \mathrm{~S}$ & $\mathrm{C}_{48} \mathrm{H}_{28} \mathrm{~N}_{8} \mathrm{O}_{8} \mathrm{~S}_{2} \mathrm{Zn}_{2}$ & $\mathrm{C}_{96} \mathrm{H}_{58} \mathrm{Co}_{4} \mathrm{~N}_{16} \mathrm{O}_{17} \mathrm{~S}_{4}$ & $\mathrm{C}_{40} \mathrm{H}_{32} \mathrm{~N}_{8} \mathrm{NiO}_{8} \mathrm{~S}_{2}$ \\
\hline Formula weight & 602.88 & 1039.64 & 2071.54 & 875.57 \\
\hline Crystal system & Orthorhombic & Triclinic & Orthorhombic & Monoclinic \\
\hline Space group & $P c c a$ & $P-1$ & $P c c 2$ & $P 2(1) / c$ \\
\hline a $(\AA)$ & 16.085 & $9.4340(8)$ & $18.396(4)$ & $10.314(4)$ \\
\hline b $(\AA)$ & $16.0849(13)$ & $12.8307(11)$ & $15.810(4)$ & $7.447(3)$ \\
\hline c $(\AA)$ & $20.2460(17)$ & $20.1416(17)$ & $17.018(4)$ & $24.040(9)$ \\
\hline$\beta($ deg $)$ & 90 & $78.8490(10)$ & 90.00 & $95.034(5)$ \\
\hline V $(\AA 3)$ & $5238.1(6)$ & $2379.0(4)$ & $4949(2)$ & $1839.2(12)$ \\
\hline$Z$ & 8 & 2 & 2 & 2 \\
\hline$D_{c}\left(g\right.$ cm $\left.{ }^{-3}\right)$ & 1.529 & 1.451 & 1.390 & 1.581 \\
\hline$\mu\left(\right.$ Mo Ka) $\left.(m m)^{-1}\right)$ & 0.958 & 1.158 & 0.815 & 0.710 \\
\hline$F(000)$ & 2416 & 1056 & 2108 & 904 \\
\hline Theta min-max & $2.0,28.2$ & $1.0,27.5$ & $1.1,25.00$ & $1.7,28.8$ \\
\hline Tot., Uniq. Data, & 47789,6471 & 20013,10590 & 34386,8700 & 16769,4740 \\
\hline $\mathrm{R}($ int $)$ & 0.037 & 0.064 & 0.071 & 0.048 \\
\hline Observed data & 5379 & 8090 & 7620 & 3729 \\
\hline $\mathrm{N}_{\text {ref }}, \mathrm{N}_{\text {par }}$ & 6471,337 & 10590,613 & 8700,619 & 4740,278 \\
\hline$R, w R_{2}[I>2 \sigma(I)]$ & $0.0345,0.1019$ & $0.0879,0.2600$ & $0.0482,0.1520$ & $0.0401,0.1140$ \\
\hline $\mathrm{S}$ & 1.14 & 1.81 & 1.08 & 1.3 \\
\hline $\begin{array}{l}\text { Min. and Max } \\
\text { Resd Dens }\end{array}$ & $-0.35,0.78$ & $-2.46,1.83$ & $-0.62,1.48$ & $-0.32,0.49$ \\
\hline
\end{tabular}

Table S2. Selected Bond Lengths (Å) and Angles (deg) for Compounds 1-4.

\begin{tabular}{|l|l|l|l|}
\hline \multicolumn{5}{|l|}{ Compound $\mathbf{1}$} \\
\hline $\mathrm{Cd}(1)-\mathrm{O}(1)$ & $2.268(2)$ & $\mathrm{Cd}(1)-\mathrm{O}(3) \# 5$ & $2.417(3)$ \\
\hline $\mathrm{Cd}(1)-\mathrm{O}(2) \# 4$ & $2.273(2)$ & $\mathrm{Cd}(1)-\mathrm{C}(24) \# 5$ & $2.717(3)$ \\
\hline
\end{tabular}




\begin{tabular}{|l|l|l|l|}
\hline $\mathrm{Cd}(1)-\mathrm{N}(3)$ & $2.304(2)$ & $\mathrm{O}(2)-\mathrm{Cd}(1) \# 4$ & $2.273(2)$ \\
\hline $\mathrm{Cd}(1)-\mathrm{O}(4) \# 5$ & $2.330(3)$ & $\mathrm{O}(3)-\mathrm{Cd}(1) \# 3$ & $2.417(3)$ \\
\hline $\mathrm{Cd}(1)-\mathrm{N}(4)$ & $2.334(3)$ & $\mathrm{O}(4)-\mathrm{Cd}(1) \# 3$ & $2.330(3)$ \\
\hline $\mathrm{O}(1)-\mathrm{Cd}(1)-\mathrm{O}(2) \# 4$ & $128.91(8)$ & $\mathrm{O}(2) \# 4-\mathrm{Cd}(1)-\mathrm{O}(3) \# 5$ & $139.68(8)$ \\
\hline $\mathrm{O}(1)-\mathrm{Cd}(1)-\mathrm{N}(3)$ & $86.83(9)$ & $\mathrm{N}(3)-\mathrm{Cd}(1)-\mathrm{O}(3) \# 5$ & $95.26(10)$ \\
\hline $\mathrm{O}(2) \# 4-\mathrm{Cd}(1)-\mathrm{N}(3)$ & $91.06(8)$ & $\mathrm{O}(4) \# 5-\mathrm{Cd}(1)-\mathrm{O}(3) \# 5$ & $53.95(8)$ \\
\hline $\mathrm{O}(1)-\mathrm{Cd}(1)-\mathrm{O}(4) \# 5$ & $145.08(9)$ & $\mathrm{N}(4)-\mathrm{Cd}(1)-\mathrm{O}(3) \# 5$ & $90.11(10)$ \\
\hline $\mathrm{O}(2) \# 4-\mathrm{Cd}(1)-\mathrm{O}(4) \# 5$ & $86.01(8)$ & $\mathrm{O}(1)-\mathrm{Cd}(1)-\mathrm{C}(24) \# 5$ & $118.30(9)$ \\
\hline $\mathrm{N}(3)-\mathrm{Cd}(1)-\mathrm{O}(4) \# 5$ & $93.20(10)$ & $\mathrm{O}(2) \# 4-\mathrm{Cd}(1)-\mathrm{C}(24) \# 5$ & $112.76(9)$ \\
\hline $\mathrm{O}(1)-\mathrm{Cd}(1)-\mathrm{N}(4)$ & $7.01(9) 8$ & $\mathrm{~N}(3)-\mathrm{Cd}(1)-\mathrm{C}(24) \# 5$ & $94.28(9)$ \\
\hline $\mathrm{O}(2) \# 4-\mathrm{Cd}(1)-\mathrm{N}(4)$ & $88.72(9)$ & $\mathrm{O}(4) \# 5-\mathrm{Cd}(1)-\mathrm{C}(24) \# 5$ & $26.82(9)$ \\
\hline $\mathrm{N}(3)-\mathrm{Cd}(1)-\mathrm{N}(4)$ & $171.91(9)$ & $\mathrm{N}(4)-\mathrm{Cd}(1)-\mathrm{C}(24) \# 5$ & $93.26(9)$ \\
\hline $\mathrm{O}(4) \# 5-\mathrm{Cd}(1)-\mathrm{N}(4)$ & $4.86(10) 9$ & $\mathrm{O}(3) \# 5-\mathrm{Cd}(1)-\mathrm{C}(24) \# 5$ & $27.13(9)$ \\
\hline $\mathrm{O}(1)-\mathrm{Cd}(1)-\mathrm{O}(3) \# 5$ & $91.24(8)$ & & \\
\hline
\end{tabular}

Symmetry code: \#1 $x, y-1, z$; \#2 $x, y+1, z$; \#3 $x+1 / 2, y,-z+1 ; \# 4-x, y,-z+1 / 2 ; \# 5 x-1 / 2, y,-z+1$

\begin{tabular}{|c|c|c|c|}
\hline \multicolumn{4}{|l|}{ Compound 2} \\
\hline $\mathrm{Zn}(1)-\mathrm{O}(1)$ & $2.027(3)$ & $\mathrm{Zn}(2)-\mathrm{O}(8) \# 3$ & $2.096(3)$ \\
\hline $\mathrm{Zn}(1)-\mathrm{O}(2) \# 1$ & 2.081(3) & $\mathrm{Zn}(2)-\mathrm{O}(3) \# 4$ & $2.140(3)$ \\
\hline $\mathrm{Zn}(1)-\mathrm{N}(1)$ & $2.131(4)$ & $\mathrm{Zn}(2)-\mathrm{N}(8)$ & $2.147(3)$ \\
\hline $\mathrm{Zn}(1)-\mathrm{N}(2) \# 2$ & $2.137(4)$ & $\mathrm{Zn}(2)-\mathrm{N}(7)$ & $2.151(3)$ \\
\hline $\mathrm{Zn}(1)-\mathrm{O}(6)$ & $2.176(3)$ & $\mathrm{Zn}(2)-\mathrm{O}(4) \# 4$ & $2.246(3)$ \\
\hline $\mathrm{Zn}(1)-\mathrm{O}(5)$ & $2.252(4)$ & Zn(2)-C(34)\#4 & $2.516(4)$ \\
\hline $\mathrm{Zn}(2)-\mathrm{O}(7)$ & $2.042(3)$ & $\mathrm{O}(7)-\mathrm{Zn}(2)-\mathrm{O}(8) \# 3$ & $120.69(12)$ \\
\hline $\mathrm{O}(1)-\mathrm{Zn}(1)-\mathrm{O}(2) \# 1$ & 119.32(13) & $\mathrm{O}(7)-\mathrm{Zn}(2)-\mathrm{O}(3) \# 4$ & $150.18(13)$ \\
\hline $\mathrm{O}(1)-\mathrm{Zn}(1)-\mathrm{N}(1)$ & 92.07(13) & $\mathrm{O}(8) \# 3-\mathrm{Zn}(2)-\mathrm{O}(3) \# 4$ & 89.05(12) \\
\hline $\mathrm{O}(2) \# 1-\mathrm{Zn}(1)-\mathrm{N}(1)$ & $90.21(14)$ & $\mathrm{O}(7)-\mathrm{Zn}(2)-\mathrm{N}(8)$ & 91.14(13) \\
\hline $\mathrm{O}(1)-\mathrm{Zn}(1)-\mathrm{N}(2) \# 2$ & 88.44(13) & $\mathrm{O}(8) \# 3-\mathrm{Zn}(2)-\mathrm{N}(8)$ & $85.15(14)$ \\
\hline $\mathrm{O}(2) \# 1-\mathrm{Zn}(1)-\mathrm{N}(2) \# 2$ & 86.73(14) & $\mathrm{O}(3) \# 4-\mathrm{Zn}(2)-\mathrm{N}(8)$ & 89.27(12) \\
\hline $\mathrm{N}(1)-\mathrm{Zn}(1)-\mathrm{N}(2) \# 2$ & 176.73(15) & $\mathrm{O}(7)-\mathrm{Zn}(2)-\mathrm{N}(7)$ & $92.78(13)$ \\
\hline $\mathrm{O}(1)-\mathrm{Zn}(1)-\mathrm{O}(6)$ & 151.90(13) & $\mathrm{O}(8) \# 3-\mathrm{Zn}(2)-\mathrm{N}(7)$ & $86.41(14)$ \\
\hline $\mathrm{O}(2) \# 1-\mathrm{Zn}(1)-\mathrm{O}(6)$ & $88.67(12)$ & $\mathrm{O}(3) \# 4-\mathrm{Zn}(2)-\mathrm{N}(7)$ & $91.05(13)$ \\
\hline $\mathrm{N}(1)-\mathrm{Zn}(1)-\mathrm{O}(6)$ & $90.16(13)$ & N(8)-Zn(2)-N(7) & $171.55(15)$ \\
\hline $\mathrm{N}(2) \# 2-\mathrm{Zn}(1)-\mathrm{O}(6)$ & $90.91(13)$ & $\mathrm{O}(7)-\mathrm{Zn}(2)-\mathrm{O}(4) \# 4$ & $90.19(12)$ \\
\hline $\mathrm{O}(1)-\mathrm{Zn}(1)-\mathrm{O}(5)$ & $92.38(12)$ & $\mathrm{O}(8) \# 3-\mathrm{Zn}(2)-\mathrm{O}(4) \# 4$ & $148.88(12)$ \\
\hline $\mathrm{O}(2) \# 1-\mathrm{Zn}(1)-\mathrm{O}(5)$ & 148.01(12) & $\mathrm{O}(3) \# 4-\mathrm{Zn}(2)-\mathrm{O}(4) \# 4$ & 59.99(12) \\
\hline $\mathrm{N}(1)-\mathrm{Zn}(1)-\mathrm{O}(5)$ & $92.97(14)$ & $\mathrm{N}(8)-\mathrm{Zn}(2)-\mathrm{O}(4) \# 4$ & 90.91(13) \\
\hline $\mathrm{N}(2) \# 2-\mathrm{Zn}(1)-\mathrm{O}(5)$ & $90.23(14)$ & $\mathrm{N}(7)-\mathrm{Zn}(2)-\mathrm{O}(4) \# 4$ & $96.56(14)$ \\
\hline $\mathrm{O}(6)-\mathrm{Zn}(1)-\mathrm{O}(5)$ & 59.52(12) & $\mathrm{O}(7)-\mathrm{Zn}(2)-\mathrm{C}(34) \# 4$ & $120.12(14)$ \\
\hline
\end{tabular}

Symmetry code: \#1 - $x+1,-y+1,-z+1$; \#2 $x-1, y+1, z ; \# 3-x+1,-y+1,-z ; \# 4 x, y, z-1$ 


\begin{tabular}{|l|l|l|l|}
\hline $\mathrm{Co}(1)-\mathrm{O}(3)$ & $1.999(3)$ & $\mathrm{Co}(2)-\mathrm{O}(7)$ & $2.026(3)$ \\
\hline $\mathrm{Co}(1)-\mathrm{O}(8)$ & $2.010(3)$ & $\mathrm{Co}(2)-\mathrm{O}(6)$ & $2.115(4)$ \\
\hline $\mathrm{Co}(1)-\mathrm{N}(2) \# 5$ & $2.132(4)$ & $\mathrm{Co}(2)-\mathrm{N}(3)$ & $2.163(4)$ \\
\hline $\mathrm{Co}(1)-\mathrm{O}(1)$ & $2.137(3)$ & $\mathrm{Co}(2)-\mathrm{N}(4) \# 6$ & $2.169(4)$ \\
\hline $\mathrm{Co}(1)-\mathrm{N}(1)$ & $2.156(4)$ & $\mathrm{Co}(2)-\mathrm{O}(5)$ & $2.199(4)$ \\
\hline $\mathrm{Co}(1)-\mathrm{O}(2)$ & $2.200(4)$ & $\mathrm{N}(2)-\mathrm{Co}(1) \# 6$ & $2.132(4)$ \\
\hline $\mathrm{Co}(2)-\mathrm{O}(4)$ & $2.006(3)$ & $\mathrm{N}(4)-\mathrm{Co}(2) \# 5$ & $2.169(4)$ \\
\hline $\mathrm{O}(3)-\mathrm{Co}(1)-\mathrm{O}(8)$ & $116.45(15)$ & $\mathrm{O}(4)-\mathrm{Co}(2)-\mathrm{O}(7)$ & $115.95(14)$ \\
\hline $\mathrm{O}(3)-\mathrm{Co}(1)-\mathrm{N}(2) \# 5$ & $87.92(15)$ & $\mathrm{O}(4)-\mathrm{Co}(2)-\mathrm{O}(6)$ & $154.24(14)$ \\
\hline $\mathrm{O}(8)-\mathrm{Co}(1)-\mathrm{N}(2) \# 5$ & $87.54(13)$ & $\mathrm{O}(7)-\mathrm{Co}(2)-\mathrm{O}(6)$ & $89.59(14)$ \\
\hline $\mathrm{O}(3)-\mathrm{Co}(1)-\mathrm{O}(1)$ & $90.26(14)$ & $\mathrm{O}(4)-\mathrm{Co}(2)-\mathrm{N}(3)$ & $92.59(14)$ \\
\hline $\mathrm{O}(8)-\mathrm{Co}(1)-\mathrm{O}(1)$ & $153.26(14)$ & $\mathrm{O}(7)-\mathrm{Co}(2)-\mathrm{N}(3)$ & $94.20(15)$ \\
\hline $\mathrm{N}(2) \# 5-\mathrm{Co}(1)-\mathrm{O}(1)$ & $95.03(14)$ & $\mathrm{O}(6)-\mathrm{Co}(2)-\mathrm{N}(3)$ & $88.60(14)$ \\
\hline $\mathrm{O}(3)-\mathrm{Co}(1)-\mathrm{N}(1)$ & $93.32(15)$ & $\mathrm{O}(4)-\mathrm{Co}(2)-\mathrm{N}(4) \# 6$ & $85.83(14)$ \\
\hline $\mathrm{O}(8)-\mathrm{Co}(1)-\mathrm{N}(1)$ & $90.96(13)$ & $\mathrm{O}(7)-\mathrm{Co}(2)-\mathrm{N}(4) \# 6$ & $87.11(16)$ \\
\hline $\mathrm{N}(2) \# 5-\mathrm{Co}(1)-\mathrm{N}(1)$ & $178.37(17)$ & $\mathrm{O}(6)-\mathrm{Co}(2)-\mathrm{N}(4) \# 6$ & $92.54(14)$ \\
\hline $\mathrm{O}(1)-\mathrm{Co}(1)-\mathrm{N}(1)$ & $86.03(14)$ & $\mathrm{N}(3)-\mathrm{Co}(2)-\mathrm{N}(4) \# 6$ & $178.28(17)$ \\
\hline $\mathrm{O}(3)-\mathrm{Co}(1)-\mathrm{O}(2)$ & $150.52(13)$ & $\mathrm{O}(4)-\mathrm{Co}(2)-\mathrm{O}(5)$ & $93.25(13)$ \\
\hline $\mathrm{O}(8)-\mathrm{Co}(1)-\mathrm{O}(2)$ & $92.61(14)$ & $\mathrm{O}(7)-\mathrm{Co}(2)-\mathrm{O}(5)$ & $150.16(12)$ \\
\hline $\mathrm{N}(2) \# 5-\mathrm{Co}(1)-\mathrm{O}(2)$ & $88.54(15)$ & $\mathrm{O}(6)-\mathrm{Co}(2)-\mathrm{O}(5)$ & $61.00(13)$ \\
\hline $\mathrm{O}(1)-\mathrm{Co}(1)-\mathrm{O}(2)$ & $60.93(13)$ & $\mathrm{N}(3)-\mathrm{Co}(2)-\mathrm{O}(5)$ & $90.26(15)$ \\
\hline $\mathrm{N}(1)-\mathrm{Co}(1)-\mathrm{O}(2)$ & $90.90(16)$ & $\mathrm{N}(4) \# 6-\mathrm{Co}(2)-\mathrm{O}(5)$ & $89.15(15)$ \\
\hline & $-x+2, y)$ & \\
\hline
\end{tabular}

Symmetry code: \#1 - $x+2, y, z+1 / 2$; \#2 - $x+2, y, z-1 / 2 ; \# 3-x+1, y, z-1 / 2$; $\# 4-x+1, y, z+1 / 2 ; \# 5 x, y-1, z ; \# 6 x, y+1, z$

\begin{tabular}{|l|l|l|l|}
\hline Compound 4 & \multicolumn{5}{l|}{} \\
\hline $\mathrm{Ni}(1)-\mathrm{O}(1) \# 1$ & $2.0785(19)$ & $\mathrm{Ni}(1)-\mathrm{O}(2)$ & $2.096(2)$ \\
\hline $\mathrm{Ni}(1)-\mathrm{O}(1)$ & $2.0786(19)$ & $\mathrm{Ni}(1)-\mathrm{N}(4) \# 1$ & $2.121(2)$ \\
\hline $\mathrm{Ni}(1)-\mathrm{O}(2) \# 1$ & $2.096(2)$ & $\mathrm{Ni}(1)-\mathrm{N}(4)$ & $2.121(2)$ \\
\hline $\mathrm{O}(1) \# 1-\mathrm{Ni}(1)-\mathrm{O}(1)$ & $179.999(1)$ & $\mathrm{O}(2) \# 1-\mathrm{Ni}(1)-\mathrm{N}(4) \# 1$ & $89.60(8)$ \\
\hline $\mathrm{O}(1) \# 1-\mathrm{Ni}(1)-\mathrm{O}(2) \# 1$ & $87.36(8)$ & $\mathrm{O}(2)-\mathrm{Ni}(1)-\mathrm{N}(4) \# 1$ & $90.40(8)$ \\
\hline $\mathrm{O}(1)-\mathrm{Ni}(1)-\mathrm{O}(2) \# 1$ & $92.64(8)$ & $\mathrm{O}(1) \# 1-\mathrm{Ni}(1)-\mathrm{N}(4)$ & $92.63(8)$ \\
\hline $\mathrm{O}(1) \# 1-\mathrm{Ni}(1)-\mathrm{O}(2)$ & $92.64(8)$ & $\mathrm{O}(1)-\mathrm{Ni}(1)-\mathrm{N}(4)$ & $87.37(8)$ \\
\hline $\mathrm{O}(1)-\mathrm{Ni}(1)-\mathrm{O}(2)$ & $87.36(8)$ & $\mathrm{O}(2) \# 1-\mathrm{Ni}(1)-\mathrm{N}(4)$ & $90.40(8)$ \\
\hline $\mathrm{O}(2) \# 1-\mathrm{Ni}(1)-\mathrm{O}(2)$ & 180.0 & $\mathrm{O}(2)-\mathrm{Ni}(1)-\mathrm{N}(4)$ & $89.61(8)$ \\
\hline $\mathrm{O}(1) \# 1-\mathrm{Ni}(1)-\mathrm{N}(4) \# 1$ & $87.37(8)$ & $\mathrm{N}(4) \# 1-\mathrm{Ni}(1)-\mathrm{N}(4)$ & 180.0 \\
\hline $\mathrm{O}(1)-\mathrm{Ni}(1)-\mathrm{N}(4) \# 1$ & $92.63(8)$ & & \\
\hline
\end{tabular}

Symmetry code: $\# 1-x+2,-y+1,-z ; \# 2-x+1,-y,-z$ 


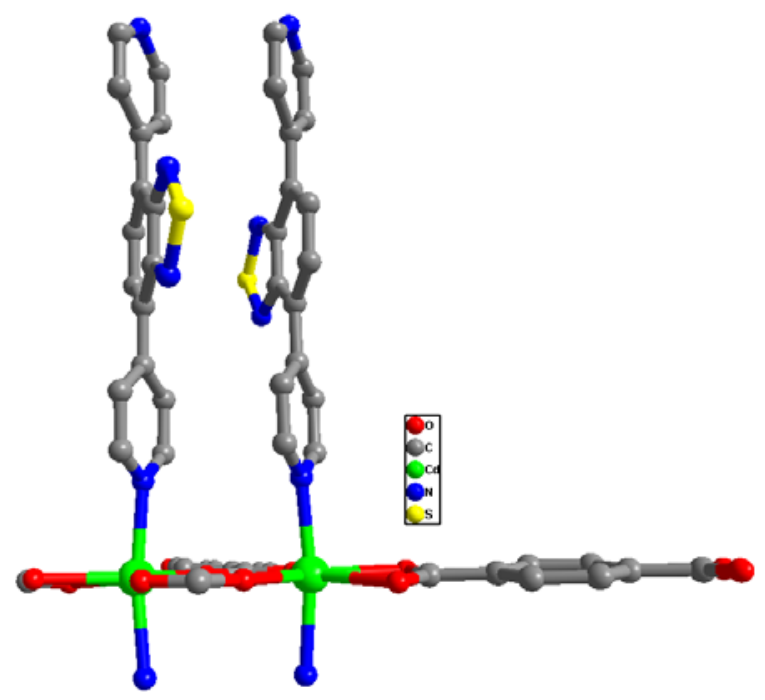

Figure S1. Coordination environment of compound 1. The hydrogen atoms are omitted for clarity.

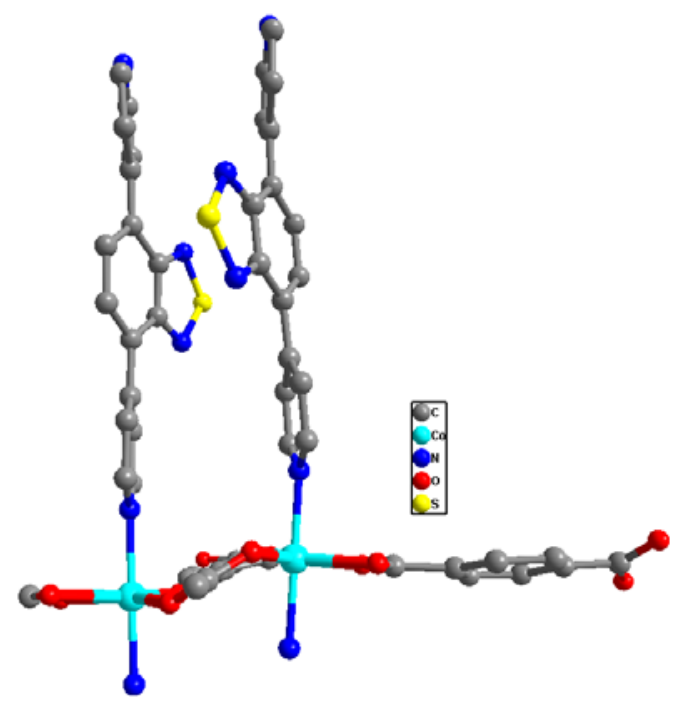

Figure S2. Coordination environment of compound 2. The hydrogen atoms are omitted for clarity. 


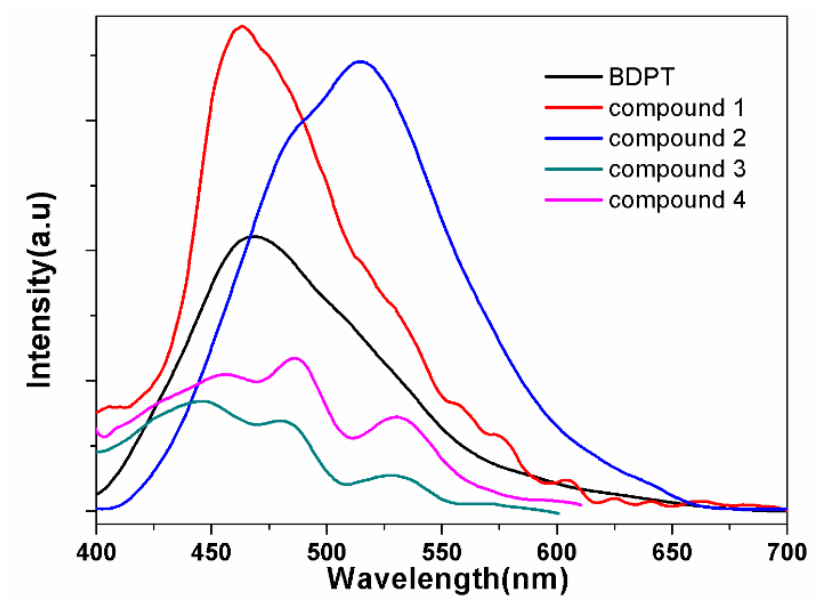

Figure S3. Solid-state emission spectra of BPTD and Compounds 1-4 $\left(\lambda_{\mathrm{ex}}=371,356\right.$, 375, 360 and 380nm, respectively).

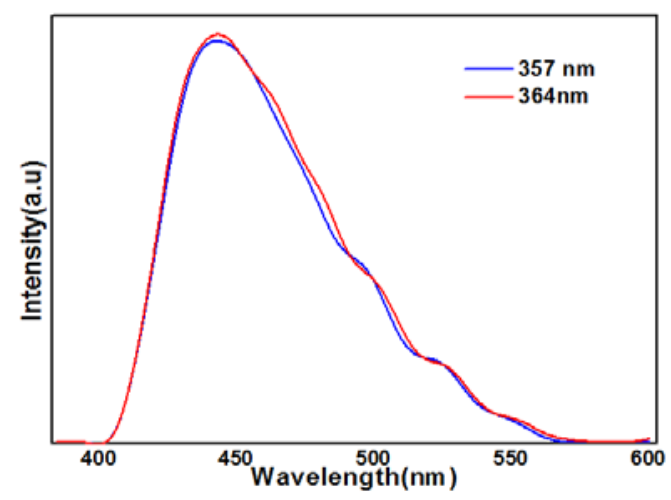

Figure S4. Liquid-state emission spectra of BPTD $\left(\lambda_{\mathrm{ex}}=357\right.$ and $\left.364 \mathrm{~nm}\right)$.

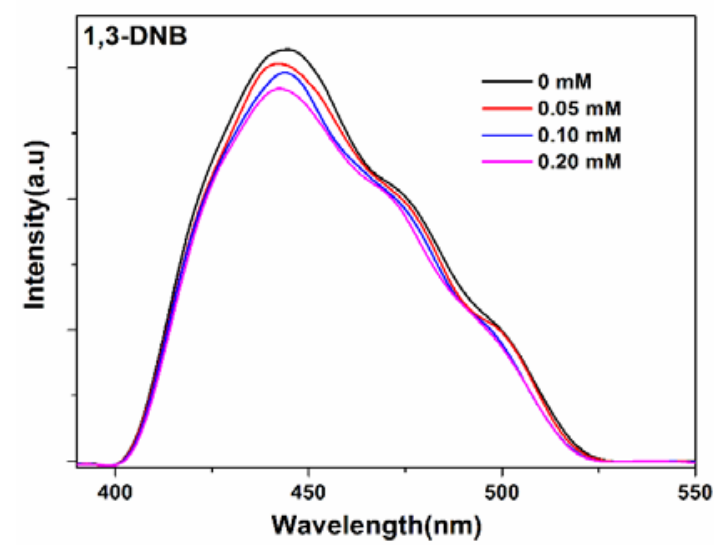

Figure S5. Concentration-dependent luminescence spectra of $\mathbf{2}$ after adding different concentrations of 1,3-DNB into DMF emulsion. 


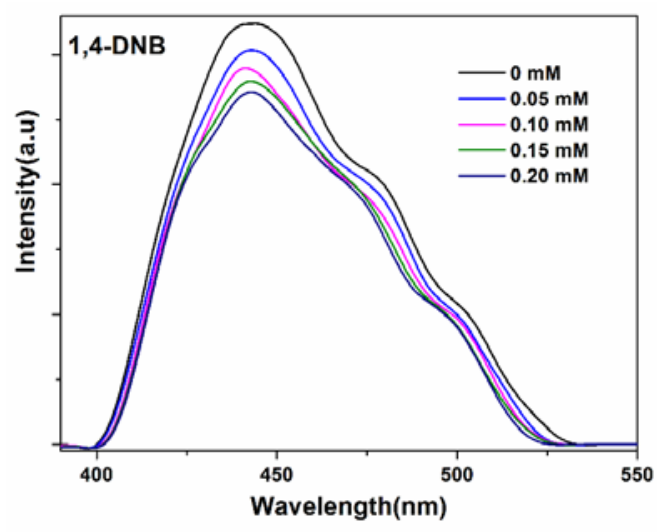

Figure S6. Concentration-dependent luminescence spectra of $\mathbf{2}$ after adding different concentrations of 1,4-DNB into DMF emulsion.

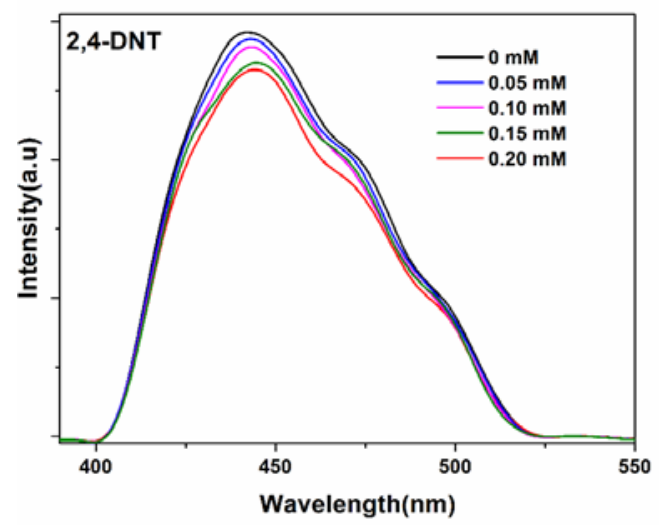

Figure S7. Concentration-dependent luminescence spectra of $\mathbf{2}$ after adding different concentrations of 2,4-DNT into DMF emulsion.

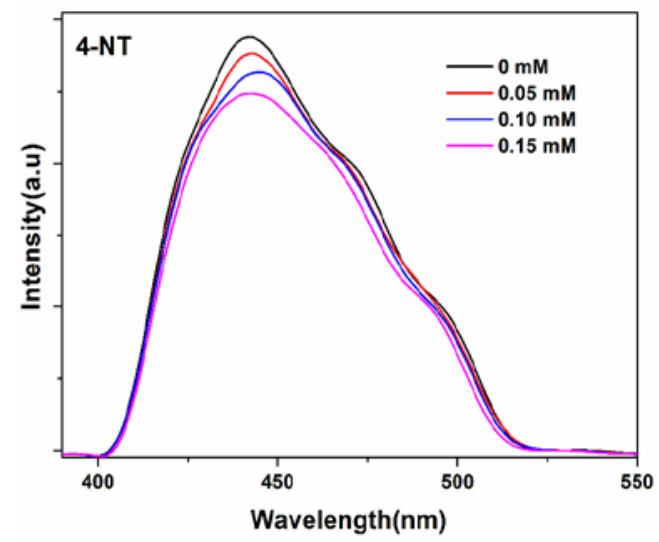

Figure S8. Concentration-dependent luminescence spectra of $\mathbf{2}$ after adding different concentrations of 4-NT into DMF emulsion. 


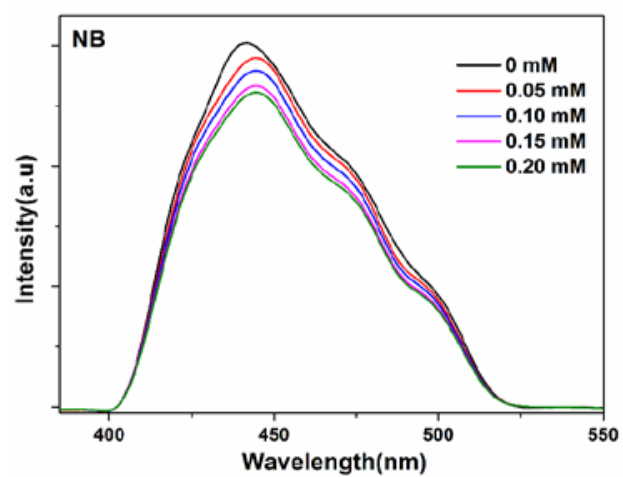

Figure S9. Concentration-dependent luminescence spectra of $\mathbf{2}$ after adding different concentrations of NB into DMF emulsion.

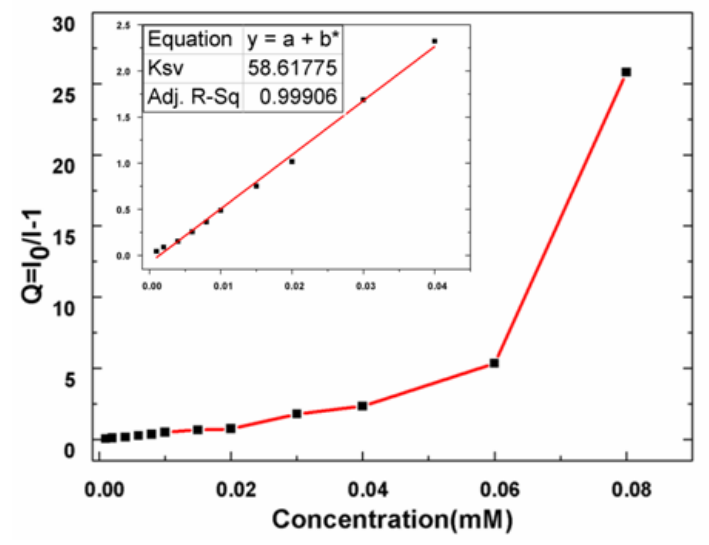

Figure S10. Stern-Volmer plot for PA.

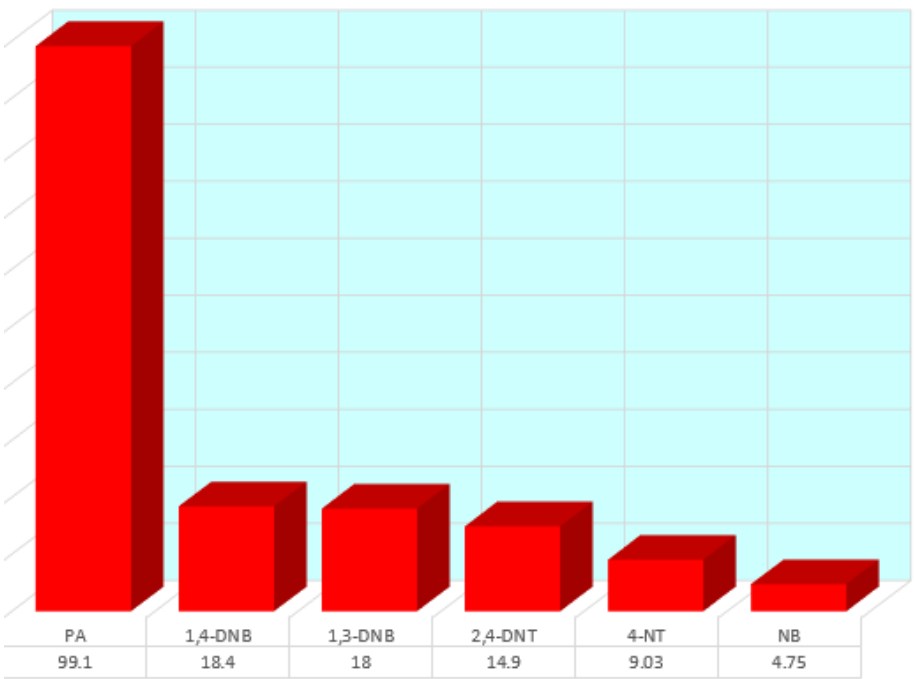

Figure S11. Luminescence quenching percentage of $\mathbf{1}$ in the presence of six different nitro compounds $(0.3 \mathrm{mM})$. 


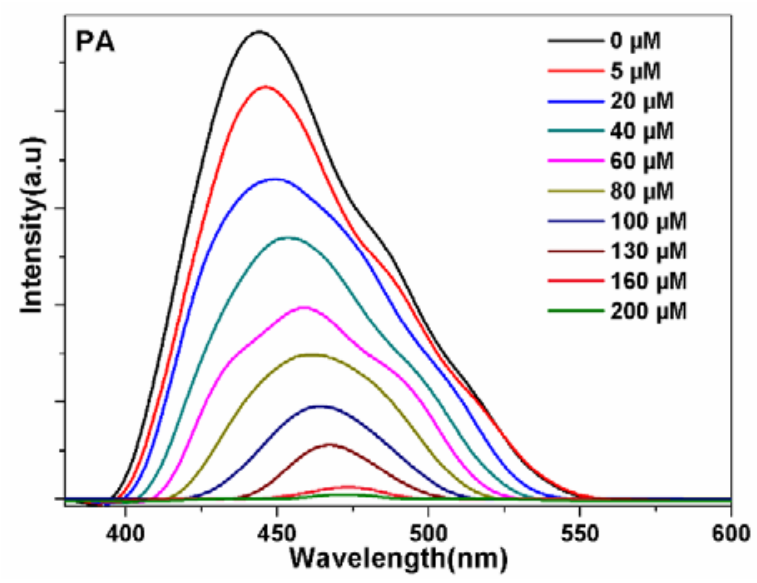

Figure S12. Concentration-dependent luminescence spectra of $\mathbf{1}$ after adding different concentrations of PA into DMF emulsion.

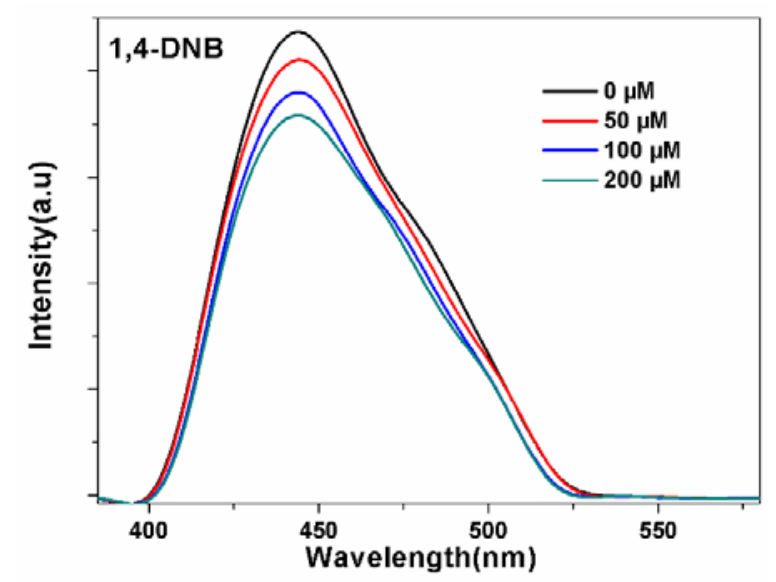

Figure S13. Concentration-dependent luminescence spectra of $\mathbf{1}$ after adding different concentrations of 1,4-DNB into DMF emulsion.

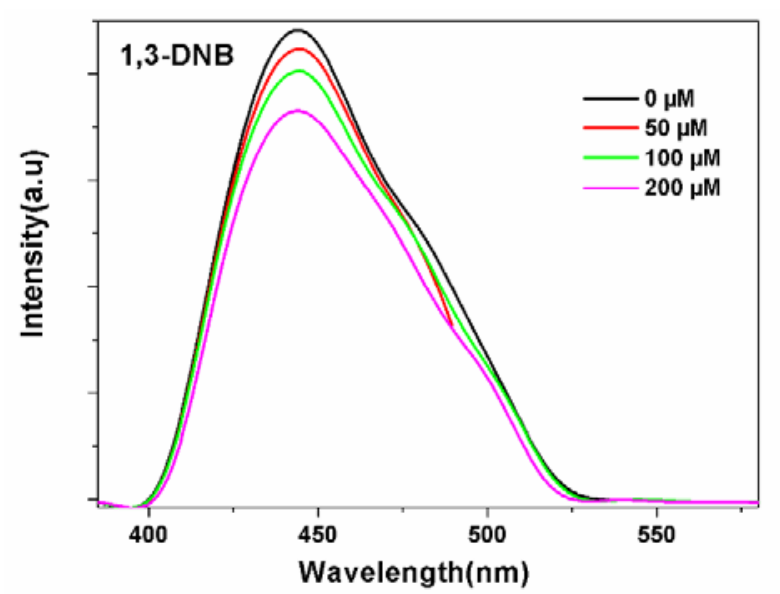

Figure S14. Concentration-dependent luminescence spectra of $\mathbf{1}$ after adding different concentrations of 1,3-DNB into DMF emulsion. 


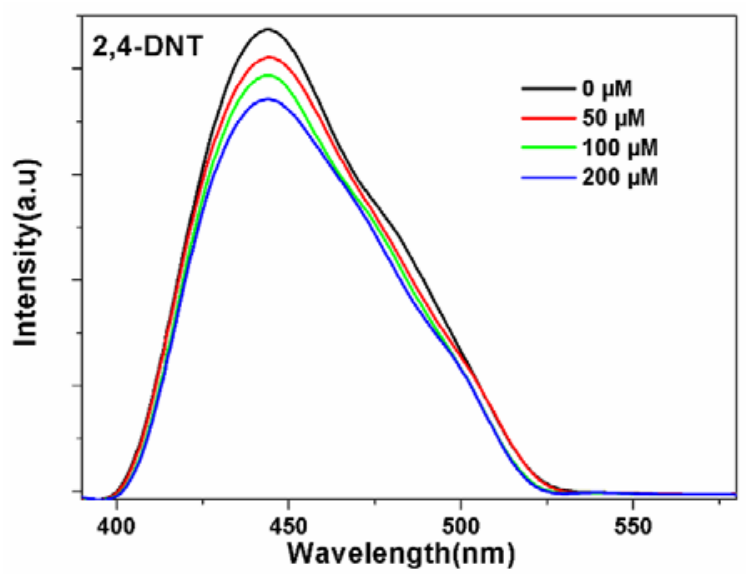

Figure S15. Concentration-dependent luminescence spectra of $\mathbf{1}$ after adding different concentrations of 2,4-DNT into DMF emulsion.

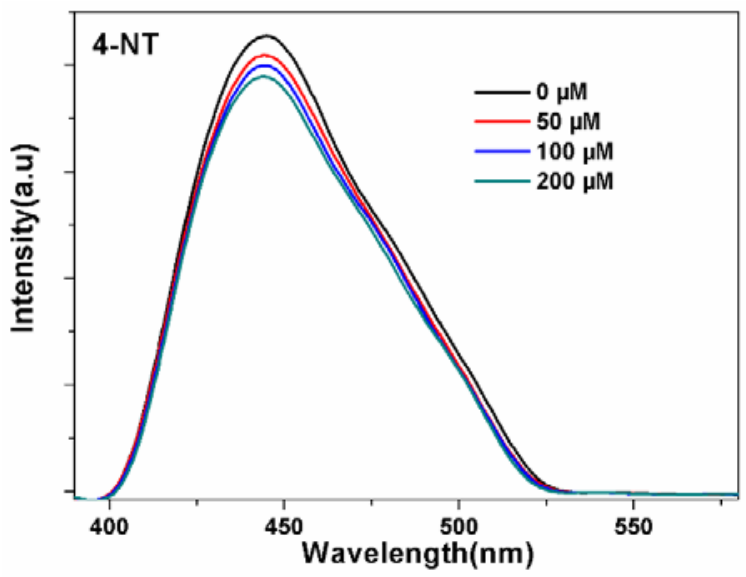

Figure S16. Concentration-dependent luminescence spectra of $\mathbf{1}$ after adding different concentrations of 4-NT into DMF emulsion.

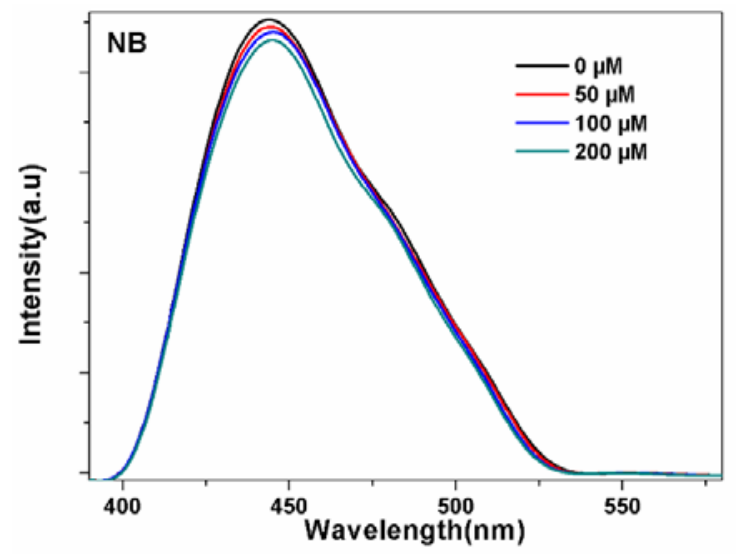

Figure S17. Concentration-dependent luminescence spectra of $\mathbf{1}$ after adding different concentrations of NB into DMF emulsion. 


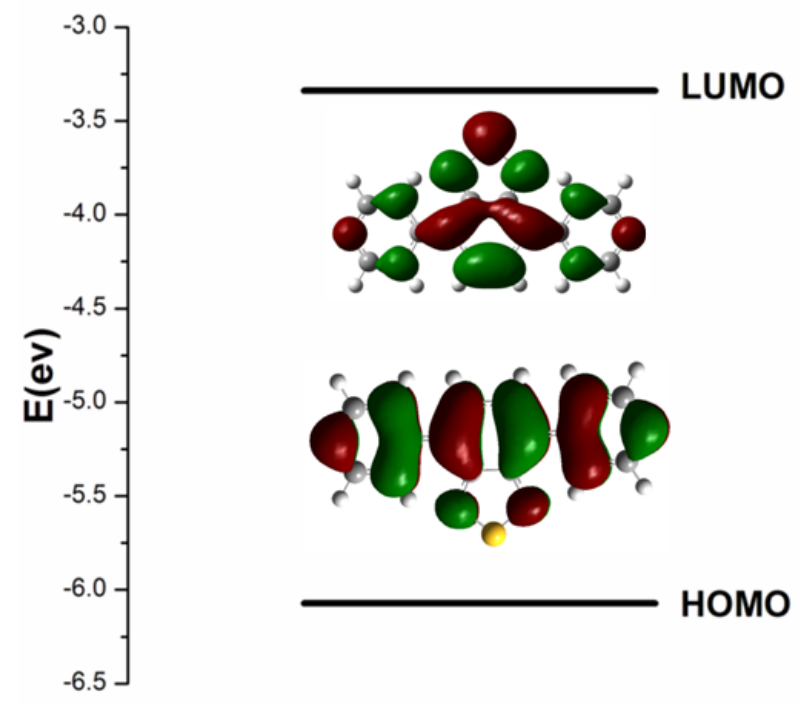

Figure S18. The HOMO and LUMO energies for DPBT ligand.

Table S3. HOMO and LUMO Levels for DPBT and Nitro Compound

\begin{tabular}{lccccccc}
\hline $\begin{array}{l}\text { DPBT and Nitro } \\
\text { Compounds }\end{array}$ & DPBT & PA & 1,4-DNB & 4-NT & $2,4-\mathrm{DNT}$ & NB & 1,3-DNB \\
\hline HOMO & -6.0726 & -8.2976 & -8.3519 & -6.9210 & -8.1181 & -7.5927 & -8.4151 \\
LUMO & -3.3765 & -3.9326 & -3.1964 & -2.2210 & -2.9877 & -2.4294 & -3.1376 \\
\hline
\end{tabular}

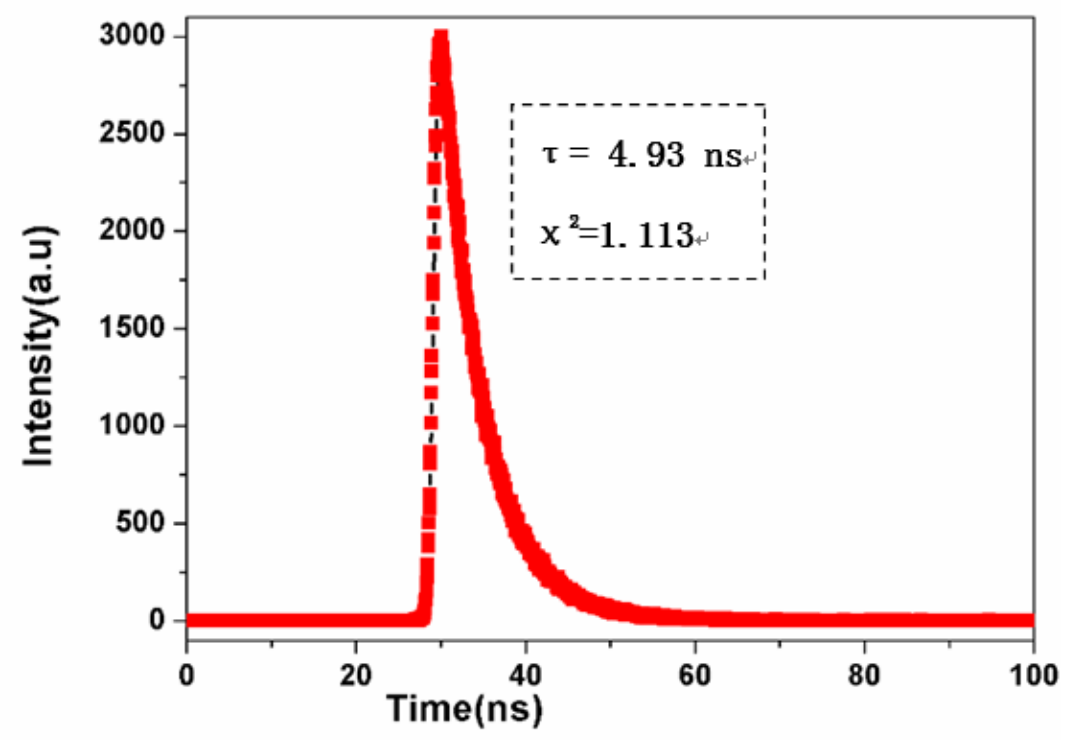

Figure S19. The fitted decay curve monitored at $443 \mathrm{~nm}$ for compound $\mathbf{1}$ in the liquid state at room temperature. The sample was excited at $357 \mathrm{~nm}$. Blank circles: experimental data; Solid line: fitted by Fit $=\mathrm{A}+\mathrm{B}_{1} \times \exp \left(-\mathrm{t} / \tau_{1}\right)+\mathrm{B}_{2} \times \exp \left(-\mathrm{t} / \tau_{2}\right)+$ $\mathrm{B}_{3} \times \exp \left(-\mathrm{t} / \tau_{3}\right)+\mathrm{B}_{4} \times \exp \left(-\mathrm{t} / \tau_{4}\right)$ 


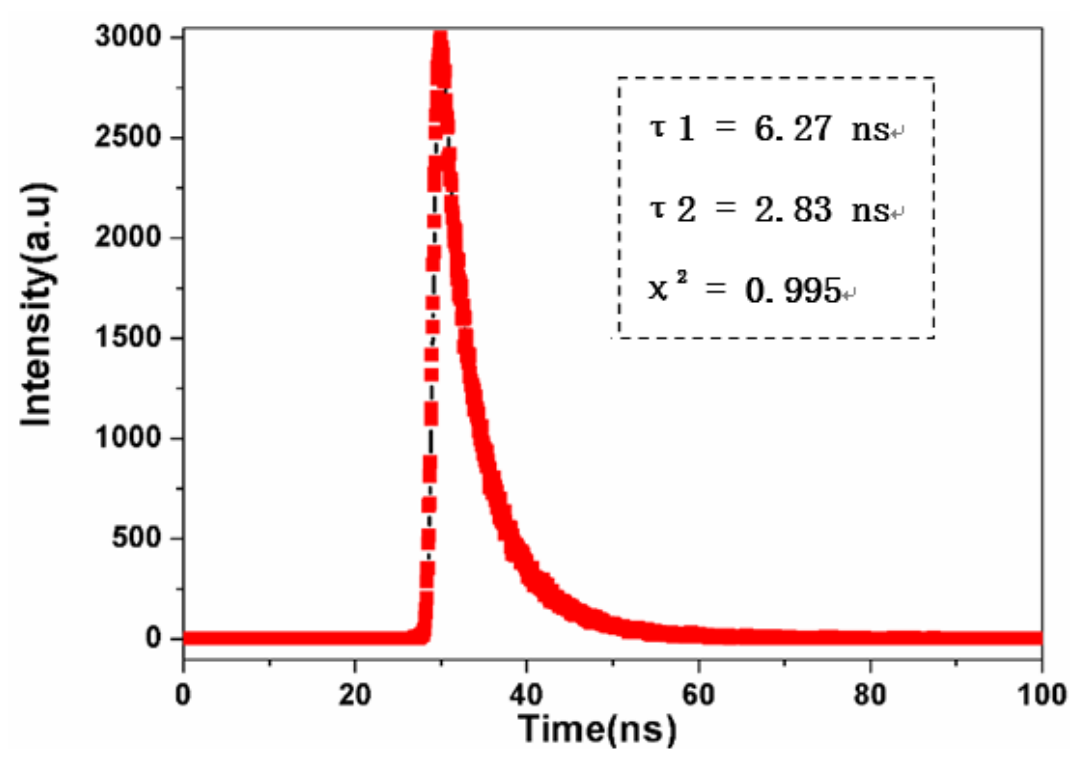

Figure S20. The fitted decay curve monitored at $443 \mathrm{~nm}$ for compound $\mathbf{1}$ in the liquid state at room temperature. The sample was excited at $364 \mathrm{~nm}$. Blank circles: experimental data; Solid line: fitted by Fit $=\mathrm{A}+\mathrm{B}_{1} \times \exp \left(-\mathrm{t} / \tau_{1}\right)+\mathrm{B}_{2} \times \exp \left(-\mathrm{t} / \tau_{2}\right)+$ $\mathrm{B}_{3} \times \exp \left(-\mathrm{t} / \tau_{3}\right)+\mathrm{B}_{4} \times \exp \left(-\mathrm{t} / \tau_{4}\right)$

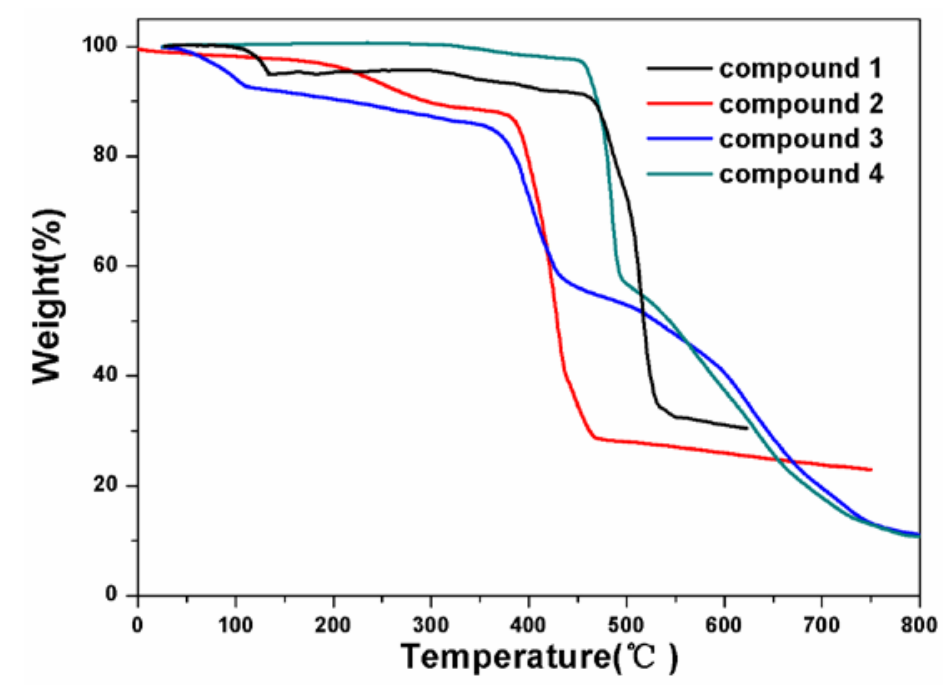

Figure S21. Thermogravimetric analysis plots of compounds 1-4. 

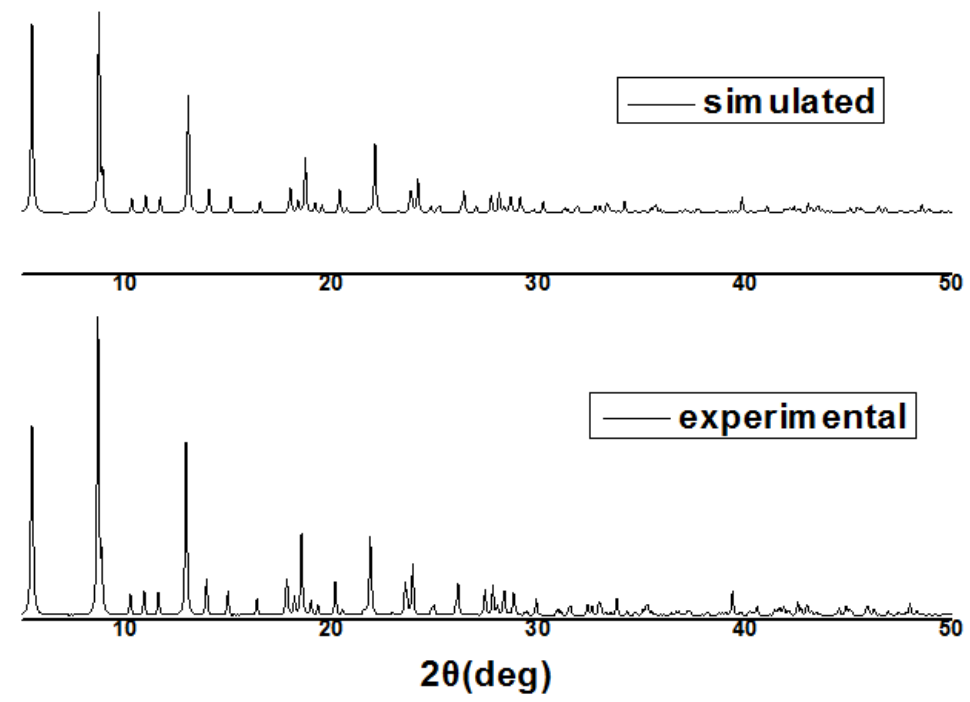

Figure S22. Powder X-ray diffraction patterns of compound 1.
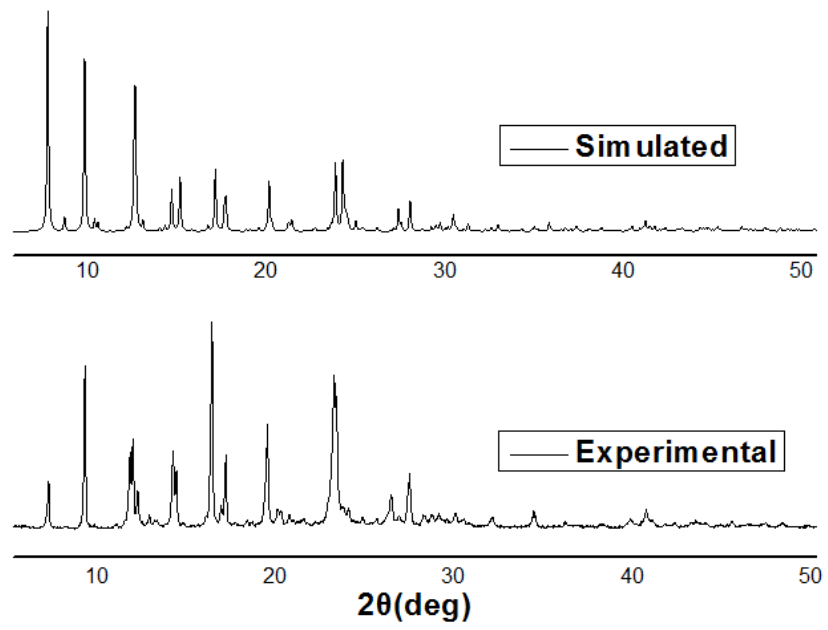

FigureS23. Powder X-ray diffraction patterns of compound 2. 


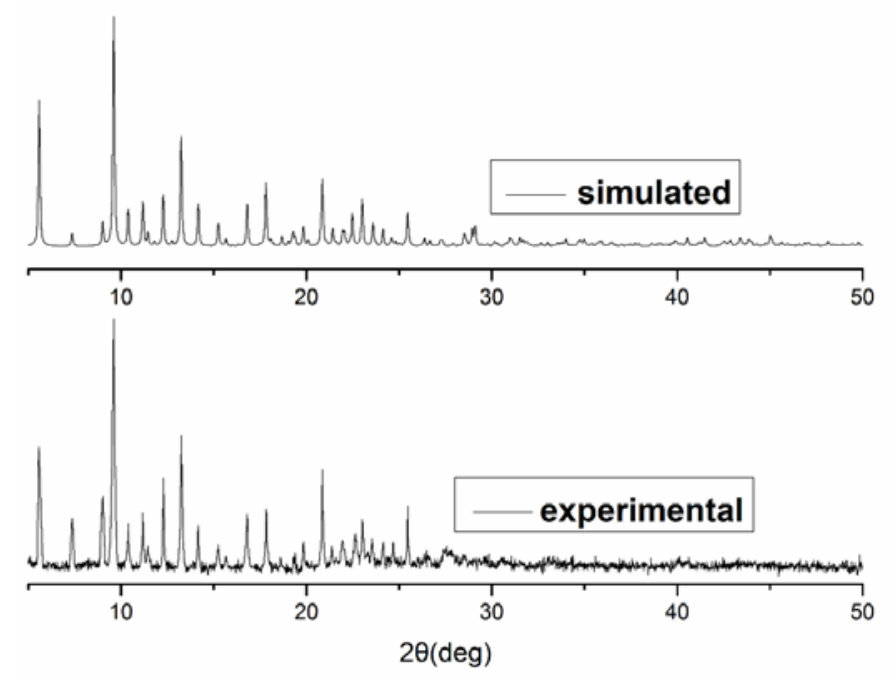

Figure S24. Powder X-ray diffraction patterns of compound 3.

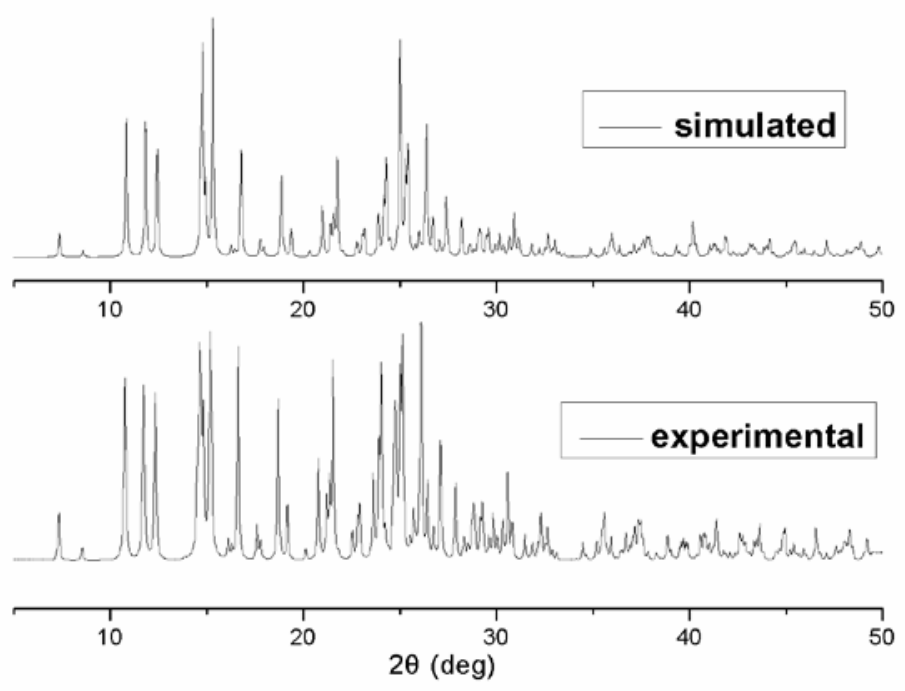

Figure S25. Powder X-ray diffraction patterns of compound 4.

\section{REFERENCES}

(1) M. J. Frisch, et al., Gaussian 09, Revision A.02, Gaussian, Inc. Wallingford, CT, 2009.Bruker 2000, SMART (Version 5.0), SAINT-plus (Version 6), SHELXTL (Version 6.1), and SADABS (Version 2.03); Bruker AXS Inc.: Madison, WI.International Tables for X-Ray Crystallography; KynochPress:Birmingham, England, 1952; Vol. III.

(2) Steware, J. J. P. J. Mol. Model. 2007, 13, 1173-1273. 
(3) M. C. Zerner, Reviews of Computational Chemistry, VCH, New York, 1991, p. 313XPREP, version 5.1; Siemens Industrial Automation Inc.: Madison, WI, 1995.

(4) Bruker 2000, SMART (Version 5.0), SAINT-plus (Version 6), SHELXTL (Version 6.1), and SADABS (Version 2.03); Bruker AXS Inc.: Madison, WI. 